\title{
Carnets
}

Revue électronique d'études françaises de l'APEF

Deuxième série - 4 | 2015

Regards sur Camus

\section{De la femme trahie à La Femme adultère: Medée et Janine ou la sensualité perdue et retrouvée}

\section{Sofia Chatzipetrou}

\section{(2) OpenEdition}

Journals

\section{Édition électronique}

URL : http://journals.openedition.org/carnets/1567

DOI : 10.4000/carnets. 1567

ISSN : 1646-7698

Éditeur

APEF

\section{Référence électronique}

Sofia Chatzipetrou, « De la femme trahie à La Femme adultère : Medée et Janine ou la sensualité perdue et retrouvée », Carnets [En ligne], Deuxième série - 4 | 2015, mis en ligne le 30 mai 2015, consulté le 30 avril 2019. URL : http://journals.openedition.org/carnets/1567 ; DOI : 10.4000/ carnets. 1567

Ce document a été généré automatiquement le 30 avril 2019.

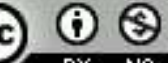

Carnets est mis à disposition selon les termes de la licence Creative Commons - Atribution - Pas d'utilisation commerciale 4.0 International. 


\title{
De la femme trahie à La Femme adultère: Medée et Janine ou la sensualité perdue et retrouvée
}

\author{
Sofia Chatzipetrou
}

1 « Tu as navigué d'une âme furieuse loin de la demeure paternelle, franchissant les doubles rochers de la mer, et tu habites une terre étrangère » (Camus, 2008, III : 608). C'est ainsi que le chœur chante le malheur de Médée dans la tragédie d'Euripide ${ }^{1}$. Mise en exergue dans Retour à Tipasa, cette citation indique l'affection que Camus éprouve à l'égard de Médée. Entre la "demeure paternelle » et la "terre étrangère », il y a une disjonction essentielle, qui est chez Camus une position fondatrice. L'œuvre est établie en effet sur la base de la dualité ; chaque notion, principe ou élément comporte son pôle opposé. L'envers présuppose son endroit. Exil-patrie, lumière-obscurité, jour-nuit, viemort, homme-femme : des schémas figurant comme conséquence d'un monde tout envahi par l'ambiguiité. Il en reste, pourtant, une antithèse majeure: celle entre le corps et l'esprit qui détermine, parmi d'autres, deux personnages de la scène tragique et de l'écriture camusienne: Médée et Janine. Partie d'une sensualité perdue et recherchée, l'existence charnelle des deux femmes définit la conscience de leur soi. Et le corps est également le sujet de la conscience : «l'existence d'un corps sentant, touchant, voyant, c'est-à-dire d'un corps sujet, est impliquée par la possibilité même de sentir, toucher, voir des corps-objets » (Legrand, 2010 : 302). L'être humain est donc une personne incarnée, un corps-image (et imagé) qui peut se contempler dans un miroir.

Donnée évidente et constitutive de l'existence, le corps et la corporéité se place au cœur de l'aventure des deux femmes. L'héroïne trahie d'Euripide et La Femme adultère de Camus sont mal aimées, étrangères et exilées d'elles-mêmes; elles s'aperçoivent de l'inaccessibilité de leur royaume, issu d'une sensualité trahie et inachevée. La nouvelle, comme on le sait déjà, est celle qui ouvre L'Exil et le Royaume, et son titre fait l'écho de l'expérience contradictoire qui fonde l'écriture camusienne. Janine, l'épouse insatisfaite d'un voyageur algérois découvre un soir, depuis une terrasse qui domine le désert, la déchirante beauté du monde et s'y livre toute entière. Médée est le drame d'une femme 
trahie, abandonnée et emportée par sa vengeance qui déchire tout ce qui lui rappelle son malheur. L'échec de la vie conjugale, la possibilité de fuir vers un ailleurs figure parmi les premiers points de leur convergence. Pour l'hérö̈ne d'Euripide, la patrie perdue indique que «l'exil entraîne bien des maux à sa suite " $(M E, 153)^{2}$; mais la nostalgie de la terre natale dispose d'un deuxième aspect symbolique : c'est aussi la distance qui la sépare d'avec le sujet aimé. Médée a quitté sa patrie pour rejoindre le royaume d'amour. Elle dit que « nul malheur n'est plus grand que d'être loin du sol natal» $(M E, 161)$ : mais elle se trouve exilée de sa deuxième patrie aussi, celle de l'amour. Dans les Fragments d'un discours amoureux, Barthes remarque que l'exil c'est la situation où « le sujet se voit avec tristesse exilé de son Imaginaire" (Barthes, 1977: 123). C'est précisément le cas de Médée ; Janine d'autre part n'avait pas songé à cet imaginaire, elle "rêvait [seulement] aux palmiers droits et flexibles, et à la jeune fille qu'elle avait été » $(E R, 9)$, selon ses mots. Si elle ne voit pas sa place dans la vie conjugale, Médée la voit détruite et perdue. Passive, Janine est en attente sensuelle sans le savoir, tandis que Médée vit en conséquence de la sensualité perdue. Janine «attendait, mais elle ne savait quoi. Elle sentait seulement sa solitude, et le froid qui la pénétrait, et un poids plus lourd à l'endroit du cœur » $(E R, 8)$.

3 Tout au contraire, Médée n'attend pas; elle agit. Le bonheur d'autrefois fait d'elle une femme « toute livrée à la douleur (...) consumée par d'éternelles larmes, depuis le jour où elle apprit qu'elle était rejetée» (ME, 135), selon le chœur. Le souvenir du passé se transforme en ruines du présent. Inversement chez Janine, la médiocrité du présent indique le malaise, le sentiment de ne pas être à sa place, la fragilité et la faiblesse. Elle se demande "pourquoi était-elle venue » $(E R, 15)$; elle est donc étrangère (comme Médée d'ailleurs), étrangère dans sa vie et dans l'espace précis de ce voyage avec son mari. Dans la nouvelle en fait, nous distinguons l'éveil progressif de sa conscience : Janine passe de l'ataraxie à l'extase. Au début de la narration, tout se passe comme si elle est absente, comme si elle n'appartient vraiment ni au paysage ni à sa vie. Toutes proportions gardées, avant que Médée apparaisse sur scène, il y a plus de deux cents vers qui décrivent son chagrin. Ainsi est mise en relief l'intensité d'une passion qui commandera la suite de l'histoire. Sorte de Caligula au féminin, Médée «ira plus loin que personne en son audace meurtrière " $(M E, 144)$. Pour elle, amour signifie donc souffrance. Et une fois sa vengeance consommée, elle apparaît à la fin de la pièce sur un char magique qui l'emporte et qui fait d'elle une absence superbe. Comme chez Martha du Malentendu, qui s'enfuit du monde "sans être réconciliée " (Camus, 2006, I : 491), la pièce d'Euripide s'achève dans la prédominance froide de Médée, « dans une haine sans pardon et sur un affrontement sans issue » (Romilly, $1986: 78$ ).

Ce qui triomphe dans Médée, c'est sa lucidité étonnante et presque choquante, l'extraordinaire intelligence de la femme qui, exilée du corps et de l'amour, est capable de tout. Le processus se réalise inversement chez Janine : l'expérience corporelle - cette extase nocturne présentée plutôt comme acte charnel - précède, et fait naître, la prise de conscience. Autrement dit, l'expérience charnelle relève la nature du soi. D'emblée, nous discernons trois étapes majeures chez elle: la perception du monde extérieur, une conscience de plus en plus lucide et, enfin, l'intégration au monde physique qui lui fait découvrir son vrai ego. Sans le savoir au début de la nouvelle, Janine est en quête d'une valeur identitaire ; et cette valeur est liée - comme au cas de Médée - à l'image et la perception corporelles qui font élucider la conscience de soi. Or, le Moi et le Moi-corps coexistent et s'entremêlent chez les deux femmes. C'est avec Platon (dans Phédon) et Descartes (avec ses Méditations métaphysiques) que s'est imposée la disjonction entre âme 
et corps, entre transcendance et immanence. Pour les deux philosophes ${ }^{3}$, âme et corps sont deux substances distinctes. Dans Les Passions de l'âme, Descartes reprend la division platonicienne du désir attaché au corps et de la volonté attachée à l'âme, sans suivre le concept d'Aristote qui ne fait aucune division de l'âme entre une partie rationnelle et une autre irrationnelle. Pour lui, il n'y a pas de conflit entre désir et raison. Chez Spinoza en plus, l'âme et le corps sont une seule et même chose, une substance unique ; dans le Traité Politique, il parle même de jouissance de l'âme. Nous considérons donc que logos (raison) et pathos (passion) s'entremêlent chez Médée et Janine et déterminent les personnages en construction à partir de leur perception charnelle.

5 «Je sais devant quel crime je me trouve mais la colère emporte mes résolutions » (ME, 183), dit l'héroïne d'Euripide. Toutefois, il y en a quelque chose à ajouter : la passion de sa vengeance. Mais comme l'affirme Camus dans ses Carnets: "vivre avec ses passions suppose qu'on les a asservies » (Camus, 2006, II : 1001). Maître et esclave de sa passion au même temps, Médée va jusqu'au bout. Avec elle en effet, Euripide décrit la force de l'irrationnel dans l'âme. La passion la transforme en victime qui souffre autant qu'elle agit : pour le poète, l'homme est conduit par des émotions passionnées qui l'entraînent vers la catastrophe qu'il n'est pas toujours capable ni de prévoir ${ }^{4}$ ni d'éviter. Camus note d'ailleurs: "aller jusqu'au bout, ce n'est pas seulement résister mais aussi se laisser aller » (Camus, 2006, II : 823). C'est précisément le cas de Janine. Au cours de la narration, elle commence à concrétiser, à connaître cet objet de manque encore inconnu : «Là-bas, plus au sud encore, à cet endroit où le ciel et la terre se rejoignaient dans une lignée pure, là-bas, lui semblait-il soudain, quelque chose l'attendait qu'elle avait ignoré jusqu'à ce jour et qui pourtant n'avait cessé de lui manquer " $(E R, 13)$. Quand elle monte sur la terrasse pour la première fois, elle a sous les yeux l'immensité de l'espace : «l'espace s'élargissait et ils s'élevaient dans une lumière de plus en plus vaste, froide et sèche, où chaque bruit de l'oasis leur parvenait avec une pureté distincte» $(E R, 12)$. Cette immensité suggère - comme le dirait Gaston Bachelard - que le « lointain est présent [et que] l'horizon a autant d'existence que le centre» (Bachelard, 1964: 184). Envahie par cette vision panoramique, Janine "perçoit là un avant-goût du mystère " (Prouteau, 2008: 220) qui indiquera, plus tard, le centre d'elle-même. L'espace vaste sert ici de prélude pour l'ouverture au monde et à soi-même.

6 Cet espace inconnu et infini est celui du silence, d'une sensualité en suspens, d'une nouvelle liberté, d'un absolu auquel Janine aspire, un sentiment de vide qui lui donne le vertige. Le ciel nocturne et sa dureté métallique ouvrent sur le désert, qui figure d'ailleurs parmi les dix mots préférés ${ }^{5}$ de Camus. Dans la nouvelle en fait, le désert a son unité géographique mais dispose, aussi, d'une symbolique d'unité : avec son «silence [qui] était vaste comme l'espace » $(E R, 13)$, Janine comprend que ce silence est une manière de communication avec elle-même, que cette terre désertique est, peut-être, sa vraie patrie. Cet « espace 'pensé" (Durand, 1992 : 476) éveille sa conscience. Ainsi, elle commence de s'apercevoir qu'elle joue un rôle dans sa vie banale, tandis que Médée joue un rôle pour parvenir à son but. À partir du moment où " ce qu'on attendait demeure inachevé » (ME, 198) selon ses propres mots, Médée se vengera d'être exilée de la vie conjugale alors que Janine veut s'en libérer. Dans la Prière d'insérer de L'Exil et le Royaume, Camus écrit :

Un seul thème, pourtant, celui d'exil, y est traité (...) quant au royaume dont il est question aussi, dans le titre, il coïncide avec une certaine vie libre et nue que nous avons à retrouver, pour renaître enfin. L'exil, à sa manière, nous en montre les chemins, à la seule condition que nous sachions y refuser en même temps la servitude et la possession. (Camus, 2008, IV : 123) 
7 C'est à cette difficile liberté que visent les deux femmes, bien que de façon différente. Les « espoirs déçus» $(M E, 154)$ de Médée et le royaume des pierres, révélateur de l'exil de Janine, suggèrent donc l'irruption de quelque chose d'inattendu qui change leur vie. «Sur moi tombe aujourd'hui un coup inattendu qui me brise et m'anéantit» (ME, 143), dit Médée : celui de la sensualité trahie. D'autre part, de la vie conventionnelle de Janine surgira un moment d'exception, une apocalypse du soi à partir de l'expérience corporelle en pleine nuit. Merleau-Ponty remarque d'ailleurs que la chair désigne une extériorité qui s'intériorise ou une intériorité qui s'extériorise. Le « schéma corporel » des deux femmes fait donc que l'image du corps soit indissociable de la conscience incarnée.

Même si les deux écrits tournent autour du personnage féminin, la présence-absence masculine y est constitutive. En réalité, c'est par rapport aux hommes que la sensualité de deux femmes se définit, se trahit et s'accomplit enfin. Dans l'extase finale de Janine, on remarque un principe puissant d'érotisme - jamais achevé dans la vie conjugale. C'est la nostalgie de l'unité amoureuse (et trahie) qui arme la main de Médée. Le "poison du désir » $(M E, 160)$ qui infeste son cœur fait d'elle une femme " audacieuse, faite de roc ou bien de fer » (ME, 190), comme la nomme le chœur. Le « grand démon »(Platon, 1991 : 87) qu'est l'amour pour le philosophe grec, suggère que, dans la tragédie, les victimes d'Aphrodite sont des femmes possédées. Tout se passe comme si l'aspect dionysiaque du rituel dramatique envoûte les femmes amoureuses, pour les soumettre à ce que le pouvoir d'Éros et d'Aphrodite peut avoir de destructeur et de mortifère. Or, la folie de Médée est, en partie, le résultat des outrances des dieux de l'amour. Selon Nietzsche cependant : « il $\mathrm{y}$ a toujours un peu de folie dans l'amour. Mais il y a toujours aussi un peu de raison dans la folie » (Nietzsche, 1983 : 56). La lucidité étonnante de Médée, manifestée dès le début de la pièce, s'oppose à la prise de conscience progressive chez Janine. Grâce à la passion amoureuse, Médée s'est exilée de sa patrie pour vivre dans le royaume de l'amour, d'où le même homme aimé l'a expulsée et condamnée à un double exil. Faute de passion amoureuse au contraire, Janine se sent exilée à cause d'un amour et d'une sensualité jamais retrouvés dans sa vie de couple. Elle "aimait être aimée » $(E R, 4)$, dit-elle. Plus tard, fascinée par la vie des nomades, elle s'aperçoit que leur mode de vie correspond à son aspiration de vivre libre, de ne rien posséder ; cette liberté lui révèle sa soif d'absolu, pareille chez Médée. Janine découvre donc sa dépendance: elle n'existe qu'en tant qu'objet de Marcel. Son mari symbolise l'exil et la servitude. Il est intéressant de remarquer que dans la chambre d'hôtel - synonyme de son exil - elle se sent mal à l'aise : "Janine sentit le froid qui venait des murs nus et blanchis [et] elle sentait seulement sa solitude » $(E R, 8)$. Le retranchement de Janine par rapport à son milieu (qu'est la chambre d'hôtel) implique une rupture avec le quotidien qui suscite l'éveil de conscience. Mais sur la terrasse de l'extase, toute livrée à la nuit, elle ne sent même pas le froid. Janine « s'ouvrait un peu plus à la nuit. Elle respirait, elle oubliait le froid, le poids des êtres, la vie démente ou figée, la longue angoisse de vivre et de mourir. Après tant d'années où, fuyant devant la peur, elle avait couru follement, sans but, elle s'arrêtait enfin » $(E R, 18)$.

Mais si Janine désire « s'arrêter ", Médée ne peut ni veut s'arrêter. Dès qu'elle apparaît, la scène devient le royaume de sa passion. Chez elle, il n'y a pas seulement colère et jalousie. Il y a dans le déchaînement de sa passion un élan si irrésistible qu'elle parait vraiment possédée. Mais sa fureur n'est pas aveugle; elle contient, surtout, une volonté de domination. Sa rage n'a pas été suscitée par la seule trahison de celui qu'elle aimait, mais par le fait qu'elle se voit réduite à rien, déchue, humiliée. Elle aspire à survivre pour savourer son triomphe. Son soi est anéanti et, dès lors, elle veut détruire tout ce qui 
appartient à Jason. L'amour profond pour lui avait changé le monde, mais Médée se trouve maintenant en plein déséquilibre; plus rien n'a de sens pour elle, même pas la maternité. La valeur existentielle de la passion définit donc le rapport entre homme et femme, puisque « ni Médée, ni Jason ne seraient eux-mêmes l'un sans l'autre » (Boulogne, 1997 : 213). Leur conjonction, constitutive de leur être, renvoie à une certaine délinéation entre le masculin et le féminin que l'on trouve chez Camus. Cette délinéation, qui définit la corporéité et la sensualité, démontre la présence discrète, parfois muette, des femmes dans son œuvre. Tout se passe comme si les femmes sont créées pour embellir le royaume masculin, mais en sont exilées en définitive. On pense ici à Victoria de L'État de Siège, Dora des Justes et, bien sûr, à Maria du Malentendu. En fait, c'est «dans le creux de leurs absences/silences que leur présence se fait essentiellement sentir » (Montgomery, 2004 : 117). C'est aussi le cas de Janine. Absente de sa vie conjugale, elle devient présente à soimême, « silencieuse devant l'étendue sans limites » $(E R, 12)$ selon ses mots. Elle s'ouvre donc à l'ampleur de la nuit, alors que Médée se livre à l'immensité du ciel. Entre dépassement et accomplissement de soi, les deux femmes s'aperçoivent que leur amour est «nostalgie du plein [pour Médée] et désir du vide [pour Janine] ( (Merrien, $2010: 17$ ); Janine était «incapable de s'arracher au vide qui s'ouvrait devant elle» $(E R, 13)$. Et comme tout être humain qui désire, elles aussi désirent ce que Platon décrit dans Le Banquet : «ce qui n'est ni présent, ni disponible, ce [qu'elles n'ont] pas, ce qu'il n'est pas, ce qui [leur] manque » (Platon, $1991: 83$ ).

10 Si donc la sensualité des deux femmes se définit à partir de la vie conjugale, Médée et Janine trouvent ce qu'elles cherchent hors de la vie conjugale : en elles-mêmes. Le désir trahi et inachevé exprime donc la conscience d'un corps qui, attribuée au soi, établit l'accomplissement et l'identification à soi-même. Dans L'Histoire de l'érotisme, Bataille souligne que le désir se présente sous la forme paradoxale d'une répulsion qui attire, sorte de déchirement qui comble et qui complète. Pour les deux femmes, la conscience corporelle dispose donc d'une valeur identitaire, d'une affirmation de soi construite à partir de la sensualité. Contrairement aux préjugés qui tiennent Euripide pour un misogyne, il conteste, avec Médée, la triste et injuste condition réservée aux femmes dans une société faite pour les hommes, par les hommes. Au fur et à mesure, c'est la même dépendance que Janine découvre par rapport à Marcel: elle n'existe qu'en tant que son objet: "à lui faire sentir si souvent qu'elle existait pour lui, il la faisait exister réellement" $(E R, 4)$, dit-elle. Mais l'union nocturne avec la nature constitue une réconciliation avec elle-même, une identification à soi-même. Contrairement à Médée, Janine regagne sa vie en tant que femme et être humain, et non comme mère, épouse, ou amante. Le personnage de Janine est en réalité l'unique exemple camusien d'autocréation féminine par elle seule et pour elle seule. Sur cette terrasse où " le ciel entier s'étendait audessus d'elle, renversée sur la terre froide » $(E R, 18)$, Janine existe, au sens propre du terme. Puisque c'est la nature qui lui relève ce mystère, il ne s'agit pas de transcendance, car à aucun moment Janine ne se sépare du monde physique. Camus s'intègre d'ailleurs à la nature pour en parler; il la décrit dans la mesure où il est sans cesse en rapport avec elle; et « comme les Grecs je crois à la nature » (Camus, 2006, II : 1066), note-t-il dans les Carnets. L'union de Janine avec la nature est donc une expérience cosmique, qui figure ensuite comme révélation. Elle découvre sa vérité. Selon Épicure en outre, de même que la vérité se reconnaît par la sensation, un sujet se reconnaît comme tel par un double critère affectif : plaisir et douleur. Il n'est pas donc étonnant que Janine dise : "en tous lieux, désormais, la vie était suspendue, sauf dans son cœur où, au même moment, quelqu'un pleurait de peine et d'émerveillement » $(E R, 14)$. 
11 La « peine » de Janine se lie aux « éternelles larmes» $(M E, 135)$ de Médée : pour toutes les deux, c'est l'exil qui les restitue à elles-mêmes. L'héroïne d'Euripide pourtant n'appartient pas aux filles dociles qui acceptent passivement la fatalité. Elle conduit son destin et, dans son rapport avec l'autre sexe, elle est en quête de parité et non pas de subordination. Transfigurée par le triomphe de sa vengeance, Médée n'est plus dominée par sa passion de puissance : elle est devenue cette puissance. Elle va jusqu'au bout de sa passion, vouée, corps et âme, au démon de l'amour. Comme Janine qui est devenue cette plénitude, livrée toute entière aux sources de la nuit et de la lune qui régénèrent son être. Dans la terre désertique, "il lui semblait retrouver ses racines » $(E R, 18)$; elle identifie donc sa vraie patrie, résumant "à elle toute seule l'univers féminin et lunaire » (Reichelberg, 1983 : 111). Dans cette nouvelle toute lunaire où le froid règne en maître, le désert traduit le repos, la plénitude et l'ataraxie que Janine recherchait. En montant vers la terrasse, elle s'élève au-dessus de l'absurdité et de sa vie stérile. Pour Janine, comme pour Meursault, il y a eu catharsis. " Elle voulait être délivrée » $(E R, 16)$, dit-elle ; et elle l'a été enfin. De tous les personnages camusiens en fait, Janine est la seule à vivre l'extase presque sacrale, sans mourir à la fin. En s'ouvrant "à la tendre indifférence du monde " (Camus, 2006, I : 213) comme Meursault, Janine appartient à elle-même. Elle fait écho de ce que Camus notait en 1939: «Je m'appartenais enfin, ne m'appartenant plus. Et j'ai fermé les yeux avec délices sur cette paix que je sentais monter avec cet univers paisible qui venait de naître, sans tyrannie, sans amour et hors de moi » (Camus, 2006, II : 875). Le mysticisme sensuel de Camus implique donc le sacré et, dans cette nouvelle, l'exil relève le royaume autant que le corps exprime l'univers, selon Leibniz. Janine « savait seulement que ce royaume, de tout temps, lui avait été promis et que jamais, pourtant, il ne serait le sien, plus jamais, sinon à ce fugitif instant » $(E R, 14)$.

Mais ce «fugitif instant » est l'instant privilégié de l'ouverture sur soi-même. Comme Sisyphe, qu'il faut imaginer heureux, Janine aussi est heureuse en ce moment de se reconnaître. C'est pourquoi, la «connaissance de l'instant créateur (...) dans le jaillissement de la conscience » (Bachelard, 1992 : 18-19) indique chez Janine « le même cheminement immobile [qui] la réunissait peu à peu à son être le plus profond » $(E R, 18)$. La Femme adultère s'accomplit dans l'immobilité, tandis que la femme trahie d'Euripide disparaît sur le char magique qui l'emporte à l'immensité du ciel. Mais dans Médée, il n'y a aucune réticence, " aucune dissonance cultivée en vue d'une harmonie finale » (Rivier, 1975 : 41). Tout comme Janine qui rentre à l'exil, mais avec la différence majeure qu'elle a perçu le goût du royaume. Absent, exilé ou perdu, le "royaume » et sa réminiscence sacrée figurent comme une vraie expérience corporelle pour les deux femmes. La "sensualité exilée », qui porte la marque de la corporéité, revient à la conscience incarnée qui les ouvre à elles-mêmes. Médée et Janine incarnent, donc, la belle formule que Camus écrivait dans Noces : ce « singulier instant (...) où le bonheur naît de l'absence d'espoir, où l'esprit trouve sa raison dans le corps » (Camus, 2006, I : 136). 


\section{BIBLIOGRAPHIE}

BACHELARD, Gaston (1992). L'Intuition de l'instant. Paris : Librairie Générale Française/Stock, « Le Livre de Poche/Biblio-essais ».

BACHELARD, Gaston (1964). La poétique de l'espace. Paris : PUF.

BARTHES, Roland (1977). Fragments d'un discours amoureux. Paris : Éditions du Seuil, « Tel quel ». BouloGNE, Jacques (1997). « Approche systémique de la mythologie grecque », in Jacques Boulogne (éd.). Les systèmes mythologiques. Villeneuve d'Ascq : Presses Universitaires de Septentrion, « Travaux et Recherches », pp. 209-234.

CAmus, Albert (2006-2008). Cuvres complètes. Paris : Gallimard, « Bibliothèque de la Pléiade », 4 vols.

DURAND, Gilbert (1992). Les structures anthropologiques de l'imaginaire. Introduction à l'archétypologie générale. Paris : Dunod.

EURIPIDE (2008). Tragédies complètes I. Paris : Gallimard, « Folio/Classique ».

GODDARD, Jean-Christophe (éd.) (2005). Le corps. Paris : Librairie Philosophique J. Vrin, « Thema/ $\Theta \varepsilon ́ \mu \alpha »$.

LEGRAND, Dorothée (2010). « Le soi corporel », in B. Andrieu (éd.). Philosophie du corps. Expériences, interactions et écologie corporelle. Paris : Librairie Philosophique J. Vrin, « Textes clés », pp. 297-310. MERRIEN, Catherine (2010). L'Amour. Paris : Eyrolles, « Petite philosophie des grandes idées ». MONTGOMERY, Geraldine E. (2004). Noces pour femme seule. Le féminin et le sacré dans l'œuvre d'Albert Camus. Amsterdam-New York : Éditions Rodopi, « Faux titre ».

NiETZSCHE, Friedrich (1983). Ainsi parlait Zarathoustra. Paris : Librairie Générale Française, « Le Livre de Poche/Les Classiques de Poche ».

Platon (1991). Le Banquet. Paris : Librairie Générale Française, « Le Livre de Poche/Les Classiques de la Philosophie ».

Prouteau, Anne (2008). Albert Camus ou le présent impérissable. Paris : Orizons chez L'Harmattan, «Universités - Domaine littéraire ».

REICHELBERG, Ruth (1983). Albert Camus. Une approche du sacré. Paris : Nizet.

RIVIER, André (1975). Essai sur le tragique d'Euripide. Paris : Éditions de Boccard.

ROMILlY, Jacqueline de (1986). La modernité d'Euripide. Paris : PUF, « Écrivains ».

\section{NOTES}

1. En 1952, Camus note dans ses Carnets : "Médée - par le groupe du théâtre Antique. Je ne peux entendre ce langage sans pleurer, comme celui qui retrouve enfin sa patrie. Ces paroles sont les miennes, les miens ces sentiments, la mienne cette croyance (...) Je suis sans cité » (Camus, 2008, IV : 1131). 
2. Afin d'éviter les répétitions au long de l'article, l'abbréviation $M E$ renvoie à Médée d'Euripide et ER à L'Exil et le Royaume de Camus.

3. Dans Phèdre et La République, Platon développe la conception tripartite de l'âme ( $\psi v x \eta ́$ - psychè).

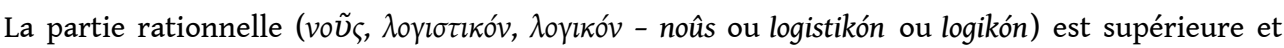
destinée à maîtriser les autres ; la deuxième partie ( $\theta v \mu o ́ s, \theta v \mu l \kappa o ́ v, \theta v \mu o \varepsilon l \delta \varepsilon ́ \varsigma$ - thumós ou thumikón ou thumoeidés) engendre le cœur, la volonté et les sentiments; liée à la jouissance, la partie

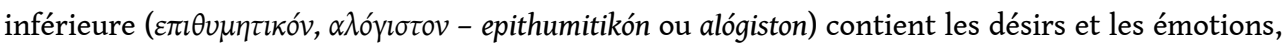
c'est l'élan qui pousse à désirer et à agir. L'équilibre psychique se préserve si la raison domine les désirs ; mais selon le philosophe, le désir est à maîtriser et non à supprimer.

4. Camus dit à ce propos: « Euripide déséquilibrera au contraire la balance tragique dans le sens de l'individu et de la psychologie. Il annonce ainsi le drame individualiste, c'est-à-dire la décadence de la tragédie » (Camus, 2008, III : 1123).

5. « Réponse à la question sur mes dix mots préférés : 'Le monde, la douleur, la terre, la mère, les hommes, le désert, l'honneur, la misère, l'été, la mer'« (Camus, 2008, IV : 1107).

\section{RÉSUMÉS}

Cette étude vise à présenter l'enjeu de la sensualité dans La Femme adultère de Camus et Médée d'Euripide. Mal aimées, étrangères et exilées d'elles-mêmes, les deux femmes s'aperçoivent de l'inaccessibilité de leur royaume, issu d'une sensualité trahie et inachevée. Si Janine ne voit pas sa place dans la vie conjugale, Médée la voit détruite et perdue à jamais. Passive, Janine est en attente sensuelle tandis que Médée - passionnée et active - vit en conséquence de la sensualité perdue. Dans la nouvelle, on distingue l'éveil progressif de la conscience: Janine passe de l'accalmie à l'extase. L'union nocturne se présente comme acte charnel, mais aussi comme expérience métaphysique qui, ensuite, figure comme véritable révélation. Partie d'une conscience lucide, Médée se venge d'être expulsée de la vie conjugale alors que Janine y retourne : elle porte la « pierre » de son destin et la sorcière l'utilise pour exorciser son chagrin. Même si les deux écrits tournent autour de l'action féminine, la présence-absence masculine y est omniprésente. En réalité, c'est par rapport aux hommes que la sensualité de deux femmes se définit, se trahit et s'accomplit enfin.

This study aims to highlight the issue of sensuality in Albert Camus' short story The Adulterous Woman and Euripides' tragedy Medea. Unloved, strangers and exiled from themselves, the two women realize that their kingdom is inaccessible due to a betrayed and incomplete sensuality. Janine cannot find a real place in her marital life, while Medea suffers because of a destroyed marriage. Waiting for sensuality, Janine is rather passive in contrast to Medea: dynamic and passionate, she lives with the consequence of lost sensuality. In the short story, the gradual arousal of consciousness is obvious : Janine goes from calm to ecstasy. The overnight fusion is presented not only as a real copulation but as a metaphysical experience as well, which appears as revelation later on. Under the light of clear consciousness, Medea takes revenge for being expelled from her marriage, while Janine returns there: she bears the "rock" of her destiny, while Medea is using to exorcise her grief. Although both writings narrate the female action, the male presence-absence is everywhere. Indeed, both women's sensuality is defined, betrayed and finally accomplished in relation to men. 
INDEX

Keywords : Medea, Janine, sensuality, exile, reception

Mots-clés : Médée, Janine, sensualité, exil, réception

\section{AUTEUR}

SOFIA CHATZIPETROU

Un. Sorbonne Nouvelle - Paris 3

sophie.chatz[at]gmail.com 